\title{
Discrete Pulse Transform of Images
}

\author{
Roumen Anguelov and Inger Fabris-Rotelli* \\ Department of Mathematics and Applied Mathematics \\ University of Pretoria \\ Pretoria 0002, South Africa \\ roumen. anguelov@up.ac.za, \\ inger.fabris-rotelli@up.ac.za
}

\begin{abstract}
The Discrete Pulse Transform (DPT) of images is defined by using a new class of LULU operators on multidimensional arrays. This transform generalizes the DPT of sequences and replicates its essential properties, e.g. total variation preservation. Furthermore, the discrete pulses in the transform capture the contrast in the original image on the boundary of their supports. Since images are perceived via the contrast between neighbour pixels, the DPT may be a convenient new tool for image analysis.
\end{abstract}

Keywords: LULU, discrete pulse transform, total variation preservation.

\section{Introduction}

One of the powerful ideas resulting from the theory of the LULU operators for sequences, developed by Carl Rohwer during the last two decades or so, is the Nonlinear Multiresolution Analysis based on a Discrete Pulse Transform (DPT), 8]. A DPT maps a bi-infinite sequence $\xi=\left(\ldots, \xi_{-1}, \xi_{0}, \xi_{1}, \xi_{2}, \ldots\right)$ onto an infinite vector

$$
\operatorname{DPT}(\xi)=\left(D_{1}(\xi), D_{2}(\xi), \ldots\right)
$$

where $D_{n}(\xi)$ is a sequence consisting of well separated discrete block pulses with support $n$. In a recent work by the authors the definition of the LULU operators was extended from sequences to multidimensional arrays, namely, functions on $\mathbb{Z}^{d}, d>1$. This development opened the opportunity of developing the DPT for functions on $\mathbb{Z}^{d}$ by using iterative application of the generalized LULU operators, similar to the one-dimensional case. In this paper we consider the DPT of images. Let us denote by $\mathcal{A}\left(\mathbb{Z}^{2}\right)$ the set of all functions defined on $\mathbb{Z}^{2}$ which have finite support. A grey scale image is a function $f \in \mathcal{A}\left(\mathbb{Z}^{2}\right)$ such that the support of $f$ is a finite rectangular subset $\Omega$ of $\mathbb{Z}^{2}$. The DPT of a function $f \in \mathcal{A}\left(\mathbb{Z}^{2}\right)$ is a vector of the form

$$
\operatorname{DPT}(f)=\left(D_{1}(f), D_{2}(f), \ldots, D_{N}(f)\right)
$$

which is finite due to the finite support of $f$. Here $N$ is the number of pixels in the image and $D_{n}(f)=\sum_{s=1}^{\gamma(n)} \phi_{n s}$, the functions $\phi_{n s}, s=1, \ldots, \gamma(n)$, being discrete 
pulses with support of size $n, n=1, \ldots, N$. In this context a discrete pulse is a function $\phi \in \mathcal{A}\left(\mathbb{Z}^{2}\right)$ which is zero everywhere except on a connected set $V$, where it is constant. The set $V$ is called the support of the pulse $\phi$ and we write $V=\operatorname{supp}(\phi)$. The value of $\phi$ on $V$ is shortly called the value of the pulse. If the value of $\phi$ is positive then $\phi$ is an up-pulse, if it is negative, $\phi$ is a down-pulse. Via the DPT we represent a function $f \in \mathcal{A}\left(\mathbb{Z}^{2}\right)$ as a sum of pulses

$$
f=\sum_{n=1}^{N} D_{n}(f)=\sum_{n=1}^{N} \sum_{s=1}^{\gamma(n)} \phi_{n s}
$$

where the supports of the pulses are either disjoint or nested. Furthermore, we show that, similar to the DPT of sequences, the decomposition (3) preserves the total variation of $f$, that is, we have

$$
T V(f)=\sum_{n=1}^{N} T V\left(D_{n}(f)\right)=\sum_{n=1}^{N} \sum_{s=1}^{\gamma(n)} T V\left(\phi_{n s}\right) .
$$

The total variation is an important characteristic of an image and has been successfully used in noise removal procedures, e.g. [3], 13. The equalities in (4) show that the decomposition (3) does not create artificial variation, i.e. noise. We should further remark that the pulses in (3) have also a more direct meaning, namely, the contrast in the original image at the boundary of the support of any pulse is at least as much as the value of that pulse.

\section{LULU Theory}

The LULU operators on multidimensional arrays are defined in [1] by using the morphological concept of connection, 15,16], which characterizes the connected subsets of the domain. The set of connected sets appropriate for images is usually defined via a neighbor relation on the domain. More precisely, a set $C \subseteq \mathbb{Z}^{2}$ is called connected if for any two pixels $p, q \in C$ there exists a set of pixels $\left\{p_{1}, p_{2}, \ldots, p_{k}\right\} \subseteq C$ such that each pixel is neighbour to the next one, $p$ is neighbour to $p_{1}$ and $p_{k}$ is neighbour to $q$. It was shown in [1] that if the neighbor relation is reflexive, symmetric, shift invariant and includes the pairs of consecutive points both vertically and horizontally, then the collection $\mathcal{C}$ of all connected sets on $\mathbb{Z}^{2}$ is a morphological connection suitable for the LULU operators. The neighbour relations typically considered for images, e.g. as in Figure 1] satisfy these basic requirements.

Given a point $x \in \mathbb{Z}^{2}$ and $n \in \mathbb{N}$ we denote by $\mathcal{N}_{n}(x)$ the set of all connected sets of size $n+1$ which contain a point $x$, that is,

$$
\mathcal{N}_{n}(x)=\{V \in \mathcal{C}: x \in V, \operatorname{card}(V)=n+1\}
$$

Then the basic LULU operators are defined as follows. 

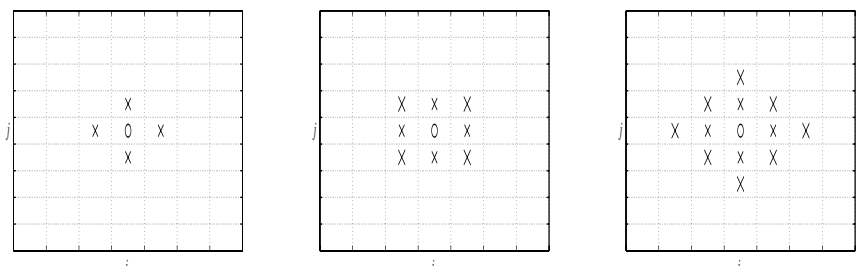

Fig. 1. Neighbours of the pixel $(i, j)$

Definition 1. Let $f \in \mathcal{A}\left(\mathbb{Z}^{2}\right)$ and $n \in \mathbb{N}$. Then

$$
\begin{aligned}
& L_{n}(f)(x)=\max _{V \in \mathcal{N}_{n}(x)} \min _{y \in V} f(y), x \in \mathbb{Z}^{2}, \\
& U_{n}(f)(x)=\min _{V \in \mathcal{N}_{n}(x)} \max _{y \in V} f(y), x \in \mathbb{Z}^{2} .
\end{aligned}
$$

The operators in Definition 1 were given in [1] as a generalization of the operators $L_{n}$ and $U_{n}$ for sequences. However, it should be noted that these are actually the well known morphological operators of area opening and area closing (section 4.4.2, [17]) considered in a new setting, namely, the LULU theory 812. It was shown in [1] that these operators are smoothers and separators (Chapter 1, 8]). Furthermore, they form, under composition, a four element totally ordered semigroup - the so called LULU semi-group [1211] - and we have

$$
L_{n} \leq U_{n} \circ L_{n} \leq L_{n} \circ U_{n} \leq U_{n}
$$

The smoothing effect of the operators, $L_{n}, U_{n}$, on a function $f \in \mathcal{A}\left(\mathbb{Z}^{2}\right)$ is described in terms of the concepts of local minimum and local maximum sets.

Definition 2. Let $V \in \mathcal{C}$. A point $x \notin V$ is called adjacent to $V$ if $V \cup\{x\} \in \mathcal{C}$. The set of all points adjacent to $V$ is denoted by $\operatorname{adj}(V)$.

Definition 3. A connected subset $V$ of $\mathbb{Z}^{2}$ is called a local maximum set of $f \in \mathcal{A}\left(Z^{d}\right)$ if

$$
\max _{y \in \operatorname{adj}(V)} f(y)<\min _{x \in V} f(x) .
$$

Similarly $V$ is a local minimum set if

$$
\min _{y \in \operatorname{adj}(V)} f(y)>\max _{x \in V} f(x)
$$

Then the action of $L_{n}$ and $U_{n}$ on $f \in \mathcal{A}\left(\mathbb{Z}^{2}\right)$ can be described in terms of the following properties, [1]:

A1 The application of $L_{n}\left(U_{n}\right)$ removes local maximum (minimum) sets of size smaller or equal to $n$. 
A2 The operator $L_{n}\left(U_{n}\right)$ does not affect the local minimum (maximum) sets directly in the sense that such sets may be affected only as a result of the removal of local maximum (minimum) sets. However, no new local minimum sets are created where there were none. This does not exclude the possibility that the action of $L_{n}\left(U_{n}\right)$ may enlarge existing local maximum (minimum) sets or join two or more local maximum (minimum) sets of $f$ into one local maximum (minimum) set of $L_{n}(f)\left(U_{n}(f)\right)$.

A3 $L_{n}(f)=f\left(U_{n}(f)=f\right)$ if and only if $f$ does not have local maximum (minimum) sets of size $n$ or less.

In analogy with the respective concept for sequences we introduce the concept of $n$-monotonicity for functions in $\mathcal{A}\left(\mathbb{Z}^{2}\right)$.

Definition 4. Function $f \in \mathcal{A}\left(\mathbb{Z}^{2}\right)$ is called (locally) n-monotone if the size of all local minimum or local maximum sets of $f$ is larger then $n$.

It follows from $\mathrm{A} 1-\mathrm{A} 3$ that for every $f \in \mathcal{A}\left(\mathbb{Z}^{2}\right)$

$$
\begin{aligned}
& \left(L_{n} \circ U_{n}\right)(f) \text { is } n \text {-monotone } \\
& \left(U_{n} \circ L_{n}\right)(f) \text { is } n \text {-monotone } \\
& \left(L_{n} \circ U_{n}\right)(f)=\left(U_{n} \circ L_{n}\right)(f)=f \Longleftrightarrow f \text { is } n \text {-monotone }
\end{aligned}
$$

\section{Discrete Pulse Transform}

The Discrete Pulse Transform (2) for a function $f \in \mathcal{A}\left(\mathbb{Z}^{2}\right)$ is obtained via iterative application of the operators $L_{n}, U_{n}$ with $n$ increasing from 1 to $N$. For a given $n$, the sequencing of $L_{n}$ and $U_{n}$ does not affect the properties of (2) stated in the Introduction. However, it introduces bias towards up-pulses or down-pulses. This issue will be given particular attention later. For now let $P_{n}$ denote either the composition $L_{n} \circ U_{n}$ or the composition $U_{n} \circ L_{n}$ and let $Q_{n}=P_{n} \circ P_{n-1} \circ \ldots \circ P_{2} \circ P_{1}$. In the general theory of Mathematical Morphology, $Q_{n}$ is known as an alternating sequential filter (see Section 8.3 [17]). However, here are interested in the portions of the image which are filtered out by the application of $P_{n}, n=1,2, \ldots, N$. Indeed, we ultimately obtain $Q_{N}(f)$, which is a constant function containing no information about the original image except possibly the general level of illumination. The rest of the information carried by $f$ is in the layers peeled off. More precisely, we have

$$
\begin{aligned}
f= & \left(i d-P_{1}\right)(f)+\left(\left(i d-P_{2}\right) \circ Q_{1}\right)(f)+\left(\left(i d-P_{3}\right) \circ Q_{2}\right)(f)+\ldots \\
& +\left(\left(i d-P_{N-1}\right) \circ Q_{N-2}\right)(f)+\left(\left(i d-P_{N}\right) \circ Q_{N-1}\right)(f)+Q_{N}(f),
\end{aligned}
$$

where $i d$ denotes the identity operator. Let us note that a similar iterative application of area opening and area closing operators is used by Acton and Mukherjee for image classification. In [2], filtering is for selected values of $n$ and instead of the layers of peeled off portions the authors keep record of filtered images at every scale (this would be $Q_{n}(f)$ in the notation adopted here). While the approach 
in [2] is adequate for the classification methods derived there, the decomposition (12) contains much more information. It is also easy to apply, not least, due to the fact that it gives a representation of $f$ in terms of discrete pulses. Indeed, we show next that (12) is a DPT decomposition of the form (3) by proving that each term is a sum of pulses of certain size. This result is obtained in the next two theorems.

Theorem 1. Let $f \in \mathcal{A}\left(\mathbb{Z}^{2}\right)$ be $(n-1)$-monotone. Then

a) $\left(i d-L_{n}\right)(f)$ is a sum of disjoint up-pulses of size $n$;

b) $\left(i d-U_{n}\right)(f)$ is a sum of disjoint down-pulses of size $n$;

c) $\left(i d-U_{n} \circ L_{n}\right)(f)$ is a sum of disjoint pulses of size $n$;

d) $\left(i d-L_{n} \circ U_{n}\right)(f)$ is a sum of disjoint pulses of size $n$.

Proof. a) Consider $x \in \mathbb{Z}^{2}$ such that $\left(i d-L_{n}\right)(f)(x)>0$. By property A1 there exists a local maximum set $V$ of $f$ such that $x \in V$ and $\operatorname{card}(V) \leq n$. Since $f$ is $(n-1)$-monotone it does not have local maximum sets of size smaller then $n$. Therefore $\operatorname{card}(V)=n$ and $f$ is constant on $V$. Moreover, we have (id$\left.L_{n}\right)(f)(y)=0, y \in \operatorname{adj}(V)$. Indeed, if $\left(i d-L_{n}\right)(f)(y)>0$ for some $y \in \operatorname{adj}(V)$ then $y$ belongs to a local maximum set $W$ of $f$ and $\operatorname{card}(W) \leq n$. However, any local maximum set containing $y$ must contain $V$ as well. Thus, $\operatorname{card}(W) \geq n+1$. This is a contradiction. So we have that the support of $\left(i d-L_{n}\right)(f)$ is a union of disjoint connected sets of size $n$, i.e.

$$
\operatorname{supp}\left(\left(i d-L_{n}\right)(f)\right)=V_{1} \cup V_{2} \cup \ldots \cup V_{\rho(n)},
$$

where $V_{s} \in \mathcal{C}$ and $\operatorname{card}\left(V_{s}\right)=n$ for $s=1,2, \ldots, \rho(n)$ and $V_{s_{1}} \cap V_{s_{2}}=\emptyset$ for $s_{1} \neq s_{2}$. Let $\left(i d-L_{n}\right)(f)(x)=\alpha_{s}$ for $x \in V_{s}$. Then

$$
\left(i d-L_{n}\right)(f)=\sum_{s=1}^{\rho(n)} \phi_{n s},
$$

where $\phi_{n s}$ is pulse with support $V_{s}$ and value $\alpha_{s}$.

The proof of b) is similar. The proof of c) follows from a) and b) by using that $i d-L_{n} \circ U_{n}=i d-L_{n}+\left(i d-U_{n}\right) \circ L_{n}$. Similar argument holds for d).

Theorem 2. Let the operators $P_{n}$ and $Q_{n}$ be as in (12). Then for every $n=$ $1, \ldots, N$ we have that $D_{n}(f)=\left(\left(i d-P_{n}\right) \circ Q_{n-1}\right)(f)$ is a sum of pulses of the form

$$
D_{n}(f)=\sum_{s=1}^{\gamma(n)} \phi_{n s},
$$

where $\operatorname{card}\left(\operatorname{supp}\left(\phi_{n s}\right)\right)=n$ and $\operatorname{supp}\left(\phi_{n s_{1}}\right) \cap \operatorname{supp}\left(\phi_{n s_{2}}\right)=\emptyset$ for $s_{1} \neq s_{2}$. Moreover, the supports of all pulses $\phi_{n s}, s=1, \ldots, \gamma(n), n=1, \ldots, N$ are either disjoint or nested, that is,

$$
\left(\operatorname{supp}\left(\phi_{n_{1} s_{1}}\right) \cap \operatorname{supp}\left(\phi_{n_{2} s_{2}}\right) \neq \emptyset, n_{1} \leq n_{2}\right) \Longrightarrow \operatorname{supp}\left(\phi_{n_{1} s_{1}}\right) \subseteq \operatorname{supp}\left(\phi_{n_{2} s_{2}}\right) .
$$


Proof. Let $n \in\{2, \ldots, N\}$. The function $Q_{n-1}(f)=P_{n-1}\left(Q_{n-2}(f)\right)$ is $(n-1)$ monotone due to (9)-(10). Then it follows from Theorem 1 that $\left(i d-P_{n}\right) Q_{n-1}(f)$ is a sum of pulses of size $n$. For $n=1$ the function $f$ is trivially 1-monotone and hence $\left(i d-P_{1}\right)(f)$ is a sum of pulses of size 1 . Note that here we may have $P_{n}=U_{n} \circ L_{n}$ and obtain the result by item c) in Theorem 1, or use item d) if $P_{n}=L_{n} \circ U_{n}$. Due to (9)-(10) the sequencing of $L_{k}$ and $U_{k}$ in the operator $P_{k}$ for $k<n$ also does not matter. Hence it remains to prove (13).

Let $\operatorname{supp}\left(\phi_{n_{1} s_{1}}\right) \cap \operatorname{supp}\left(\phi_{n_{2} s_{2}}\right) \neq \emptyset$. It follows from the construction of the pulses in the proof of Theorem[1 that the functions $Q_{n}(f)$ and $P_{n+1}\left(Q_{n}(f)\right), n \geq$ $n_{1}$, are constants on the $\operatorname{set} \operatorname{supp}\left(\phi_{n_{1} s_{1}}\right)$. Furthermore, the set $\operatorname{supp}\left(\phi_{n_{2} s_{2}}\right)$ is a local maximum set or a local minimum set of either $Q_{n_{2}-1}(f)$ or $P_{n_{2}}\left(Q_{n_{2}-1}(f)\right)$. Then by the definition of local maximum and local minimum sets it follows that $\operatorname{supp}\left(\phi_{n_{1} s_{1}}\right) \subset \operatorname{supp}\left(\phi_{n_{2} s_{2}}\right)$.

As mentioned earlier the sequencing of the operators $L_{n}$ and $U_{n}$ in $P_{n}$ results in bias towards up-pulses or down-pulses. The operator $F_{n}$, called a floor operator given by $F_{n}=P_{n} \circ P_{n-1} \circ \ldots \circ P_{2} \circ P_{1}$ where $P_{n}=U_{n} \circ L_{n}$, is biased towards uppulse. This means that for any particular size the up-pulses are extracted before the down pulses. Similarly the operator $C_{n}$, called a ceiling operator given by $C_{n}=P_{n} \circ P_{n-1} \circ \ldots \circ P_{2} \circ P_{1}$ where $P_{n}=L_{n} \circ U_{n}$, is biased towards down-pulses. A DPT using $F_{n}$ will generally have more up-pulses and fewer down-pulses compared to DPT using $C_{n}$. It is important to realize that the bias extremes will differ for different original images due to their size and contrast so interpretation should be relative to the current image.

The alternating bias separators $Z_{n}^{+}$and $Z_{n}^{-}$(see [4] and references therein) are given by:

$$
\begin{aligned}
& Z_{n+1}^{-}=\left\{\begin{array}{l}
L_{n+1} U_{n+1} Z_{n}^{-} \text {if } n \text { is even } \\
U_{n+1} L_{n+1} Z_{n}^{-} \text {if } n \text { is odd }
\end{array}\right. \\
& Z_{n+1}^{+}=\left\{\begin{array}{l}
L_{n+1} U_{n+1} Z_{n}^{+} \text {if } n \text { is odd } \\
U_{n+1} L_{n+1} Z_{n}^{+} \text {if } n \text { is even }
\end{array}\right.
\end{aligned}
$$

Using the order in the LULU semi-group (8) it is easy to see that

$$
F_{n} \leq Z_{n}^{ \pm} \leq C_{n}
$$

We should note that if $f \in \mathcal{A}\left(\mathbb{Z}^{2}\right)$ is such that $F_{n}=C_{n}, n=1, \ldots, N$, then $f$ has a unique DPT decomposition of the form (12).

Finally we discuss the total variation preservation property (44). There are several different concepts used for total variation of a function of multidimensional argument in literature. For a function $f \in \mathcal{A}\left(\mathbb{Z}^{2}\right)$ we define the total variation as

$$
T V(f)=\sum_{i=-\infty}^{\infty} \sum_{j=-\infty}^{\infty}(|f(i+1, j)-f(i, j)|+|f(i, j+1)-f(i, j)|) .
$$

It was proved in 1 that the operators $L_{n}$ and $U_{n}$ are total variation preserving. Furthermore, the composition of total variation preserving operators is also total variation preserving. Therefore the decomposition (3) obtained via (12) preserves the total variation, i.e. (4) holds. 
Table 1. Bias Percentages $\left(\times 10^{-3}\right)$ between $F_{n}, C_{n}, Z_{n}^{+}$and $Z_{n}^{-}$

\begin{tabular}{c||c|c|c|c|} 
& $C_{n}$ & $F_{n}$ & $Z_{n}^{+}$ & $Z_{n}^{-}$ \\
\hline \hline$C_{n}$ & 0 & 0.2609 & 0.2269 & 0.0340 \\
\hline$F_{n}$ & 0.2609 & 0 & 0.0340 & 0.2269 \\
\hline$Z_{n}^{+}$ & 0.2269 & 0.0340 & 0 & 0.2610 \\
\hline$Z_{n}^{-}$ & 0.0340 & 0.2269 & 0.2610 & 0 \\
\hline
\end{tabular}

The decomposition of the original image can be done using $F_{n}, C_{n}, Z_{n}^{+}$or $Z_{n}^{-}$. While it is possible that these lead to different results it is seldom significantly observable in practice. For example, the percentage bias between the operators, when applied to the image (a) in Figure 2, is given in Table 1.

\section{Illustrative Examples}

\subsection{A Partial Reconstruction}

Consider image (a) in Figure 2. We wish to extract the boat as a feature and remove the background water. We consider any feature not part of the boat as

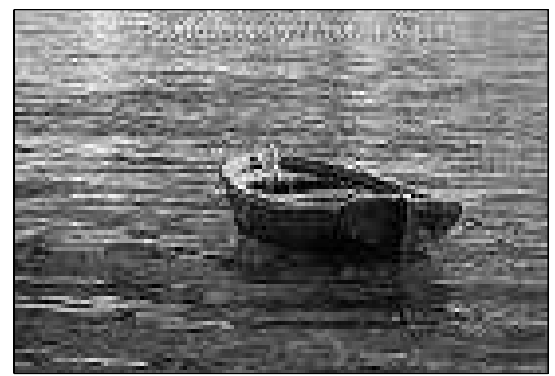

(a)

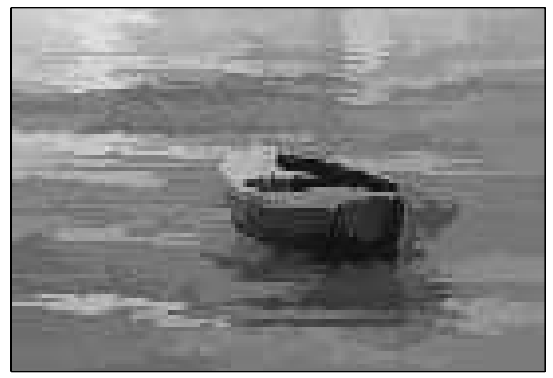

(c)

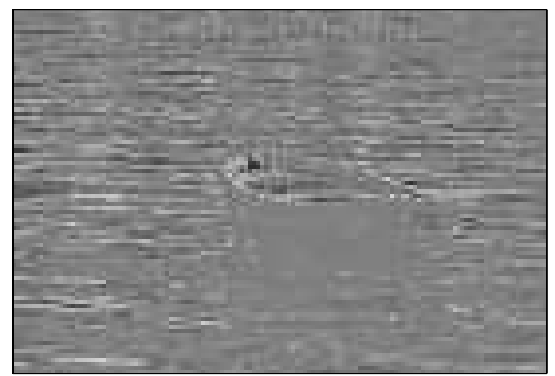

(b)

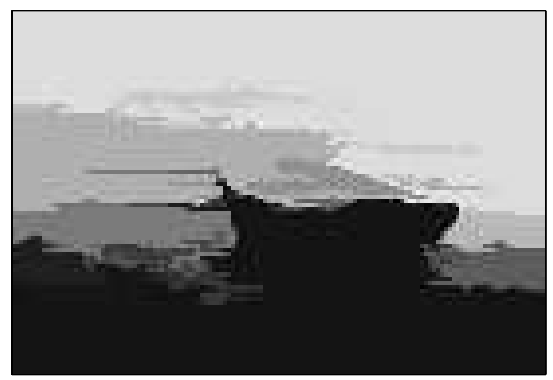

(d)

Fig. 2. (a) Original image; (b) Pulses of size up to 80; (c) Pulses of size from 81 to 4000; (d) Pulses of size larger than 4000 
noise. The images (b) - (d) in Figure 2 are partial reconstructions, see [1], of the original. We can consider (b) as the microfeatures e.g. noise, (c) as the mediumsized features and (d) as the large features. We see that pulses of size up to 80 represent the water, pulses from 81 to 4000 the boat and pulses larger than 4000 represent larger noise patterns.

\subsection{Identifying Pulses of Interest}

In Figure 3 the pulses of size 100 to 300 with relative luminosity greater than 1 represent the solar glint on the waves (see image (b) in Figure 3) and they are emphasized in image (c) by giving them all black borders. So we are able to extract the small solar glint patches from the image and can subsequently reconstruct the image without them. The pulses of size 1501 to 2500 are the pulses corresponding to the boat (as seen in image (d) of Figure 3).

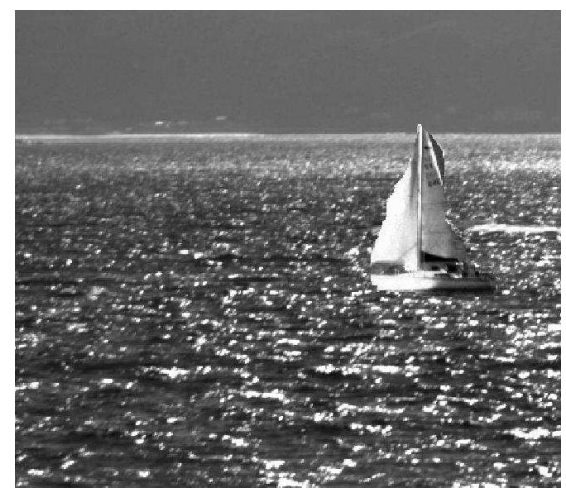

(a)

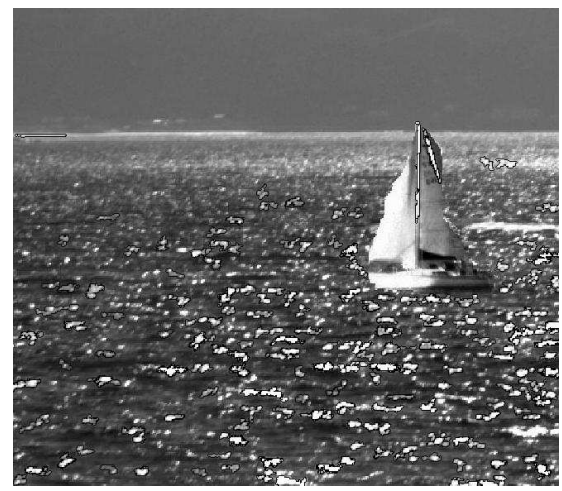

(c)

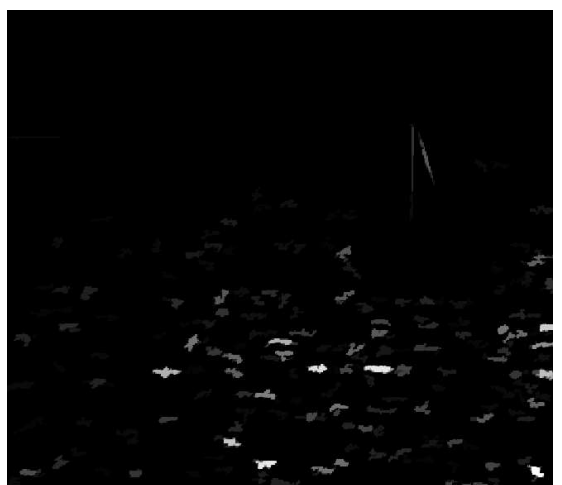

(b)

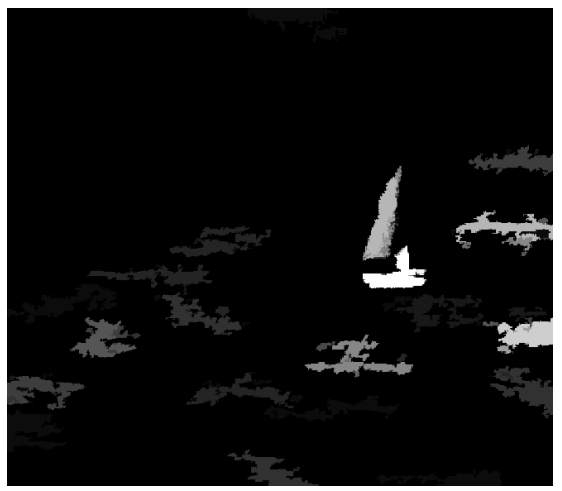

(d)

Fig. 3. (a) Original image; (b) Pulses of size 100 to 300 with relative luminosity greater than 1; (c) Borders of the pulses of size 100 to 300 with relative luminosity greater than 1 superimposed on the original image; (d) Pulses of size larger than 1501 to 2500 


\section{References}

1. Anguelov, R., Plaskitt, I.: A Class of LULU Operators on Multi-Dimensional Arrays, Technical Report UPWT2007/22, University of Pretoria (2008), http://arxiv.org/abs/0712.2923

2. Acton, S.T., Mukherjee, D.P.: Scale Space Classification Using Area Morphology. IEEE TRansactions on Image Processing 9(4) (2000)

3. Chambolle, A., Lions, P.-L.: Image recovery via total variation minimization and related problems. Numerische Mathematik 76, 167-188 (1997)

4. du Toit, J.P.: The Discrete Pulse Transform and Applications, Masters Thesis, Univeristy of Stellenbosch (2007)

5. Epp, S.S.: Discrete Mathematics with Applications. International Thomson Publishing (1995)

6. Laurie, D.P., Rohwer, C.H.: The discrete pulse transform. SIAM J. Math. Anal. 38(3) (2007)

7. Matheron, G.: Filters and lattices, in Image Analysis and Mathematical Morphology. In: Serra, J. (ed.) Theoretical Advances, ch. 6, vol. II, pp. 115-140. Academic Press, London (1988)

8. Rohwer, C.H.: Nonlinear Smoothing and Multiresolution Analysis, Birkhäuser (2000)

9. Rohwer, C.H.: Variation reduction and LULU-smoothing. Quaestiones Mathematicae 25, 163-176 (2002)

10. Rohwer, C.H.: Fully trend preserving operators. Quaestiones Mathematicae 27, 217-230 (2004)

11. Rohwer, C.H., Wild, M.: Natural Alternatives for One Dimensional Median Filtering. Quaestiones Mathematicae 25, 135-162 (2002)

12. Rohwer, C.H., Wild, M.: LULU Theory, Idempotent Stack Filters, and the Mathematics of Vision of Marr. Advances in imaging and electron physics 146, 57-162 (2007)

13. Rudin, L.I., Osher, S., Fatemi, E.: Nonlinear total variation based noise removal algorithms. Physica D 60, 259-268 (1992)

14. Serra, J.: Image Analysis and Mathematical Morphology. Academic Press, London (1982)

15. Serra, J.: Image Analysis and Mathematical Morphology. In: Serra, J. (ed.) Theoretical Advances, vol. II. Academic Press, London (1988)

16. Serra, J.: A lattice approach to image segmentation. Journal of Mathematical Imaging and Vision 24, 83-130 (2006)

17. Soille, P.: Morphological Image Analysis. Springer, Heidelberg (1999) 